\title{
Effects of different desensitizing treatments on root dentin permeability
}

Raydsa Raíssa Moura ROSA ${ }^{(a)}$ Francielle Karoline Santos CALAZANS ${ }^{(a)}$

Ruchele Dias NOGUEIRA(a)

Ailla Carla Rocha Acosta LANCELLOTTI(b)

Luciano de Sousa GONÇALVES(a)

Vinícius Rangel

GERALDO-MARTINS ${ }^{(a)}$

(a) Universidade de Uberaba, School of

Dentistry, Uberaba, MG, Brazil.

(b) Universidade Federal do Rio Grande do Sul - UFGRS, School of Dentistry, Porto Alegre, RS, Brazil.

Declaration of Interests: The authors certify that they have no commercial or associative interest that represents a conflict of interest in connection with the manuscript.

Corresponding Author:

Prof. Dr. Vinicius Rangel Geraldo Martins

E-mail:vinicius.martins@uniube.br

DOI: 10.1590/1807-3107BOR-2016.vol30.0111

Submitted: Oct 01, 2015

Accepted for publication: Jun 13, 2016

Last revision: Jul 28, 2016
Abstract: The objective of this study was to evaluate the effects of diode laser and a desensitizing dentifrice on dentin permeability. Fifty-two root dentin fragments were obtained $(5 \times 5 \mathrm{~mm})$ and treated with $24 \%$ EDTA gel. The samples were divided into 4 groups ( $n=13$ ): G1, control (no treatment); G2, diode laser $(\lambda=908 \mathrm{~nm}, 1.5 \mathrm{~W}$, continuous mode, 20s); G3, application of abrasive dentifrice for 1 minute (Elmex Sensitive Professional (International Gaba); and G4, application of abrasive dentifrice for 1 minute followed by irradiation with diode laser. Ten samples per group were immersed in $2 \%$ methylene blue solution for $4 \mathrm{~h}$. The specimens were washed, longitudinally sectioned, observed under optical microscopy, photographed and assessed based on the degree of dye leakage. The remaining samples were observed under scanning electron microscopy (SEM). The leakage data were subjected to ANOVA test, followed by Tukey's $t$-test ( $\alpha=5 \%$ ). Groups 2, 3 and 4 showed less dye penetration than the control group $(p<0.05)$, but were similar among each other. SEM images showed that dentinal tubules were open in G1, and fused and occluded in G2. Group 3 showed dentinal tubules that were occluded by the metal ions from the toothpaste. G4 presented similar characteristics to G3, and the presence of fused dentin. The diode laser and the dentifrice were effective in reducing dentinal permeability, and the combination of the two treatments did not show better results than either one used alone.

Keywords: Dentin Sensitivity; Dentin Permeability; Lasers; Dentifrices

\section{Introduction}

Dentin hypersensitivity (DH) is a dental condition characterized by a sharp pain of short duration, which cannot be attributed to any other dental pathology. This occurs when exposed dentin is affected by thermal, evaporative, tactile, osmotic, or chemical stimuli. ${ }^{1} \mathrm{DH}$ is commonly related to the loss of enamel due to abrasion, abfraction or erosion, gingival recession, or the loss of cementum due to periodontal treatment. ${ }^{2}$ People affected by DH may present alterations in chewing, swallowing, speech and nutritional habits. Female patients between the third and fourth decades of life present the highest incidence ${ }^{3}$ and the most affected teeth are the canines and pre-molars, followed by the incisors and molars. $\mathrm{DH}$ usually involve the buccal surfaces, especially the cervical area of teeth. ${ }^{2}$ The most accepted hypothesis for the occurrence of tooth sensitivity is based on the Brannström's hydrodynamic theory, which assumes that 
painful stimulation increases fluid flow within the dentinal tubules, causing the activation of local baroceptors. ${ }^{4}$ According to this theory, the sensitivity is greater in areas where there is a large amount of tubules. It is known that the density of dentinal tubules at the dentin-enamel junction is lower than in areas near the pulp chamber. ${ }^{1}$

Considering the cause of the disease, the most appropriate treatment would be the occlusion of the exposed dentinal tubules, and intratubular fluids movements inhibition. Other treatments have been reported, such as the desensitization of the pulp nerve endings, and stimulation of tertiary dentin by the pulp tissue, which reduces dentin permeability. ${ }^{2}$ As each patient responds differently to treatments, management of DH should be individualized.

The use of abrasive dentifrices is the most readily available non-invasive treatment option for patients, presenting a good cost/benefit ratio. They act by creating a smear layer over the exposed dentin, causing the precipitation of proteins, and calcium and phosphate crystals, and therefore occluding the dentinal tubules. ${ }^{5}$ The primary agent for the desensitizing effect of at-home toothpastes is potassium nitrate, which minimizes the transmission of pain impulses at the nerve endings. Recent clinical trials have reported that the control of DH may be achieved with the daily use of abrasive dentifrices over a period of 4 to 12 weeks. ${ }^{6,7}$ Studies suggest that potassium nitrate reduces sensitivity after 8 to 12 weeks if used twice a day for 5 minutes, but the effect is not longstanding. ${ }^{8}$ Toothpastes with strontium chloride and potassium citrate were also tested in in vitro and in vivo studies, obtaining satisfactory results through the partial occlusion of the dentinal tubules combined with their desensitizing action. Potassium citrate also acts by interrupting the neural response; the effects of strontium chloride are still being studied. ${ }^{9}$

The NovaMin technology is a bioactive glass in the group of highly biocompatible materials, originally developed in the 1990s as a bone regenerative material. It is composed of $25 \%$ calcium sodium phosphosilicate, $25 \%$ sodium, $25 \%$ calcium, $6.8 \%$ phosphate and silica. These materials are reactive when exposed to body fluids, causing the deposit of hydroxycarbonate apatite, a mineral chemically similar to enamel and dentin, and thus enhancing enamel remineralization. .,10 $^{-10}$

An additional therapeutic option for $\mathrm{DH}$ is the use of high and low intensity lasers. Low intensity lasers produce a rapid action with analgesic and anti-inflammatory effects, through a photobiomodulation process. ${ }^{11}$ On the other hand, high intensity lasers (Er, Cr:YSGG, $\mathrm{CO}_{2}$, Nd:YAG and diode) work on dentin via photothermal effects, heating and melting the surface of the hard tissue. When the dentin cools, it recrystallizes, thereby obliterating the dentinal tubules. Past studies have shown that the use of laser provides high desensitization durability. The $810 \mathrm{~nm}$ diode laser has been considered a satisfactory treatment modality and has a great potential in DH treatment. Diode lasers act by occluding the dentinal tubules or by reducing the pulpal nerve pain threshold..$^{12,13}$ Previous studies showed that irradiation with $980 \mathrm{~nm}$ diode laser can be effective for routine clinical treatment of $\mathrm{DH}$, due to its rapid sealing of exposed dentin tubules and its safety towards odontoblasts and pulp tissue. Additionally, high energy laser irradiation of the exposed dentin promotes a significant reduction in the dentin hydraulic conductance. ${ }^{14,15}$

Although various treatments have been proposed, none of them are considered effective in long term evaluations. Thus, it is necessary to conduct further studies to evaluate other types of treatments that might be more effective in controlling this disease. The objective of this in vitro study was to evaluate the effects of a diode laser and of a desensitizing dentifrice on dentin permeability.

\section{Methodology}

\section{Sample preparation}

The present study used freshly extracted bovine mandibular incisors. The teeth were obtained from animals slaughtered between 24 and 30 months of age. First, teeth were observed in a stereoscope and those with cracks and defects in dentin were excluded. Fifty-two freshly extracted bovine incisors were stored in a $0.1 \%$ thymol solution ( $\mathrm{pH} 7.0$ ) for one to two months. The crowns were separated from the roots using a water-cooled diamond disc (Isomet; $10.2 \mathrm{~cm} 30.3 \mathrm{~mm}$, half inch arbour size, series 15HC 
diamond; Buehler Ltd, Lake Bluff, IL, USA) mounted in a sectioning machine (Minitom, Struers Inc, Westlake, $\mathrm{OH}, \mathrm{USA})$. The roots were sectioned to obtain 52 dentin blocks $(5.0 \times 5.0 \mathrm{~mm})$. All dentin blocks were obtained from the buccal surface of incisors. The root dentin samples were ground flat and polished using water-cooled sandpaper (\#600, 800, 1200, and 2,400 grit, Saint-Gobain Abrasivos Ltda, Sao Paulo, SP, Brazil) to standardize the substrate. A $9.0 \mathrm{~mm}^{2}$ area was delimited on the surface of each sample.

\section{Experimental groups}

The dentin samples were randomly divided into four groups $(\mathrm{n}=13)$ according to Table 1 .

Prior to the experiments, a $24 \%$ EDTA (Ethylenediamine tetraacetic acid, Biodynamics Chemicals \& Pharmaceuticals Ltda., Ibiporã, PR, Brazil) gel was applied on the exposed surface of all samples. After 1 minute, each sample was cleaned in distilled water. This procedure opened the dentinal tubules, simulating a pattern of exposed dentin.

Group 1 samples were stored in distilled water throughout the experiment. The samples from group 2 were irradiated with a high intensity diode laser (SoftLase, Zap Lasers, Pleasant Hills, CA, USA), with a $908 \mathrm{~nm}$ wavelength, using a power output of $1.5 \mathrm{~W}$ (fluence $=1,194 \mathrm{~J} / \mathrm{cm}^{2}$ ) in continuous mode (non-contact). To deliver the light to the desired area, an optic fiber with a $400 \mu \mathrm{m}$ diameter was used. The root dentin surface was scanned by the laser light for a period of 20 seconds ( 10 seconds horizontally and 10 seconds vertically). After irradiation, specimens were placed in distilled water.

The samples of group 3 received a topical application of a desensitizing dentifrice (Elmex Sensitive Professional, GABA International Therwil, Switzerland), composed of arginine (8\%), sodium bicarbonate, sodium monofluorophosphate, calcium

Table 1. Experimental groups.

\begin{tabular}{lcc}
\hline Group & Treatment & $\mathrm{n}$ \\
\hline 1 (Control) & No treatment & 13 \\
2 & Diode Laser & 13 \\
3 & Dentifrice & 13 \\
4 & Dentifrice + Diode Laser & 13 \\
\hline
\end{tabular}

carbonate, water, sorbitol, sodium lauryl sulfate, sodium silicate, cellulose gum, titanium dioxide and sucralose. The dentifrice was rubbed on the dentin surface with the finger for one minute, following the manufacturer's instructions. At the end of the application, samples were washed with air/water spray to remove the excess dentifrice. This procedure was performed once in each sample.

The samples of group 4 received the same treatment as group 3, followed by the group 2 treatment. Samples were then placed in distilled water.

\section{Permeability test}

The permeability test was performed as previously described. ${ }^{16}$ After the experimental procedures, ten samples per group were randomly chosen and coated with a nail varnish (Colorama Procosa Produtos de Beleza Ltda, São Paulo/SP, Brazil) and wax (Polidental Industria e Comércio Ltda., São Paulo/SP, Brazil), except for the experimental area. Each sample was immersed in $10 \%$ copper sulfate aqueous solution (Merck, Darmstadt, Germany) for 30 minutes (the first 5 minutes in a vacuum), dried with paper, and placed in 1\% dithiooxamide alcoholic solution (Merck, Darmstadt, Germany) for 30 minutes (the first 5 minutes in a vacuum). The dithiooxamide alcoholic solution reveals copper ions, forming a compound that ranges in color from deep blue to black, depending on the quantity of copper ions present. Specimens were dried and kept in individual bottles with ammonia vapor for 7 days, for color fixation.

Subsequently, samples were individually embedded in acrylic resin and sectioned into $100 \mu \mathrm{m}$ thick sections. Then, sections were dehydrated in a series of increasing alcohol solutions $(70,80,96$, twice in $100 \%$ alcohol) for 2 hours in each solution, cleared 3 times in xylol, and mounted on glass slides for microscopic examination (Axiostar Plus, Carl Zeiss, Germany). Three sections of each specimen were observed and photographed in a light microscope, under $40 \times$ magnification. Dye penetration was measured in each image $(\mu \mathrm{m})$ using the software AxioVision LE 4.3 (Zeiss). Three sections per sample were analyzed, and an average dye penetration for each sample was calculated. 


\section{Scanning Electron Microscopy (SEM)}

For the SEM observation, three specimens of each group were randomly selected, mounted on metal stubs and sputter-coated with gold in a Denton Vacuum Desk II Sputtering device (Denton Vacuum, Cherry Hill, NJ, USA). The surfaces of the specimens were then observed under SEM (JSM-5600LV, JEOL Ltd, Tokyo, Japan) with $15 \mathrm{KV}$ at 500 and $1000 \times$ magnification.

\section{Statistical analysis}

The data were submitted to the D'Agostino normality test. One-way analysis of variance (ANOVA) and the Tukey test were used to compare the differences in dye penetration for all groups. The level of statistical significance was set at 0.05. All analyses were performed using BioEstat 5.3 (Instituto Mamiraua, Tefe, AM, Brazil).

\section{Results}

Table 2 shows the averages obtained for each group. The mean dye leakage of group $1(96.7 \pm 5.0 \mu \mathrm{m})$ was higher than the averages observed in groups 2 $(42.3 \pm 14.5 \mu \mathrm{m}), 3(44.3 \pm 9.1 \mu \mathrm{m})$ and $4(46.4 \pm 5.3 \mu \mathrm{m})$. Groups 2, 3 and 4 presented similar results.

Figure 1 presents images from groups 1 (control) and 2 (laser). The samples from group 1 (Figures 1A and B) presented no smear layer and open dentinal tubules. Samples from group 2 (Figures $1 \mathrm{C}$ and D) had an irregular dentin surface that indicated melting and resolidification. The dentinal tubules were occluded, suggesting a reduction in dentin permeability, which is in agreement with the dye leakage results.

Figure 2 presents images from groups 3 (dentifrice) and 4 (dentifrice + laser). In group 3 (Figures 2A and B), the dentinal tubules were occluded, most likely due to the deposition of minerals from the abrasive paste used. Samples from group 4 (Figures 2C and D) showed a pattern similar to that observed in group 3. However, group 4 presented a more irregular dentin surface with some micro-cracks. In both groups, the occluded dentinal tubules help to explain the results demonstrated in Table 2 .
Table 2. Comparison between dye infiltration found in each experimental group.

\begin{tabular}{lcc}
\hline Experimental groups & Treatment & Leakage $(\mu \mathrm{m})$ \\
\hline Group 1 & None & $96.7( \pm 5)^{\mathrm{a}}$ \\
Group 2 & Laser & $42.3( \pm 14.5)^{\mathrm{b}}$ \\
Group 3 & Dentifrice & $44.3( \pm 9.15)^{\mathrm{b}}$ \\
Group 4 & Dentifrice + laser & $46.4( \pm 5.37)^{\mathrm{b}}$ \\
\hline
\end{tabular}

Different superscript letters indicates a statistically significant difference.

\section{Discussion}

As described before, DH is a painful condition, most likely caused by fluid movement in the exposed dentinal tubules. ${ }^{1}$ Thus, it is expected that the occlusion of these tubules would decrease the possible fluid dynamics and dentin permeability, consequently reducing or eliminating dentin sensitivity symptoms. This study investigated the effects of a diode laser $(908 \mathrm{~nm}$ ) and a desensitizing toothpaste for reducing dentin permeability. The results showed that the two methods, or their association, were effective in reducing the penetration of dyes into the root dentin.

SEM images confirmed what was found in the dye penetration results. The samples from group 1 presented open dentinal tubules and had the highest dye infiltration. The images from groups 2, 3 and 4 show a dentin surface with occluded tubules, responsible for a low dye penetration.

The $24 \%$ EDTA gel was used on root dentin surface to remove the smear layer and expose dentinal tubules. This method is the most used in in vitro studies that evaluate treatments for $\mathrm{DH}$, because it is scientifically accepted, and it simulates a clinical condition. ${ }^{12,17,18}$ The wide use of EDTA also facilitates result comparisons among studies. In our study, SEM images showed that $24 \%$ EDTA gel was effective in removing the smear layer from the dentin surface. Other substances also used to expose dentinal tubules, especially in studies of dental erosion, include the hydrochloric acid or $6 \%$ citric acid. ${ }^{15,19}$ Hydrochloric acid is present in the oral cavity of patients with gastroesophageal reflux, and citric acid is present in the diet of patients with dental erosion. However, citric acid can react with free calcium ions, producing calcium citrate crystals, which could interact with hydroxyapatite and result in dentinal tubules obliteration. ${ }^{15}$ 

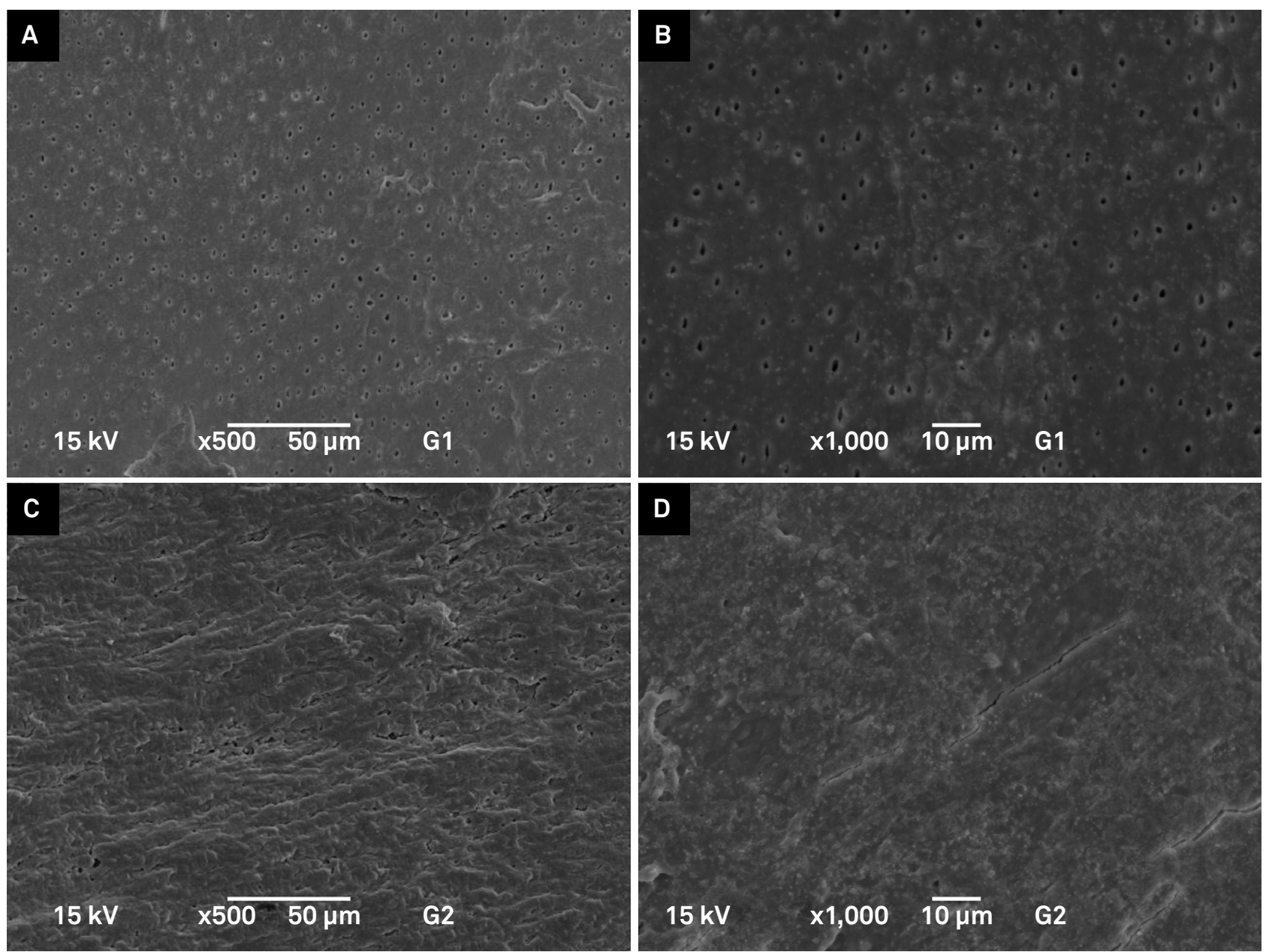

Figure 1. SEM representative image of samples from groups 1 ( $A$ and $B$ ) and 2 ( $C$ and $D$ ). Note that in $A$ and $B$, the dentinal tubules are open, while in $C$ and $D$, the tubules of the irradiated dentin are not visible. Magnification of $500 \times(A$ and $C)$ and $1000 \times(B$ and $D)$.

The present study showed that the heat generated by the high intensity diode laser was able to occlude the dentinal tubules by melting the dentin, which resolidified after cooling. The effect was a result of dentin laser irradiation with a power output of $1.5 \mathrm{~W}$ (fluence of $1,194 \mathrm{~J} / \mathrm{cm}^{2}$ ), in the absence of water cooling and without photosensitizers. Previous reports have obtained similar effects on dentin, but using lasers with higher energy densities. ${ }^{12,20}$

The laser wavelength used in the current study is well absorbed by hemoglobin and dark colored substances, but not as much by water and hydroxyapatite. Therefore, the effective use of this wavelength in hard dental tissues requires either one of the two following alternatives. The first is to increase the energy emitted by the diode laser.
However, as few molecules absorb the wavelength in the dentin surface, the risk of the laser light reaching the pulp, which is a vascularized tissue and thus has a large amount of hemoglobin, is increased. This could raise the temperature inside the pulp chamber and, in extreme cases, promote necrosis of the pulp tissue. ${ }^{21,22}$ The literature shows that irradiation of root dentin with a fluence of $3,184 \mathrm{~J} / \mathrm{cm}^{2}$ increases the intrapulpal temperature by approximately $2{ }^{\circ} \mathrm{C}$, which means that the energy density used in the present study $\left(1,194 \mathrm{~J} / \mathrm{cm}^{2}\right)$ is safe for use in vital teeth. ${ }^{20}$ The second alternative is to apply a photosensitizer on the tissue. The photosensitizer is a colored substance that can absorb the laser light on the tissue surface, decreasing the risk of temperature increase inside the pulp chamber. 

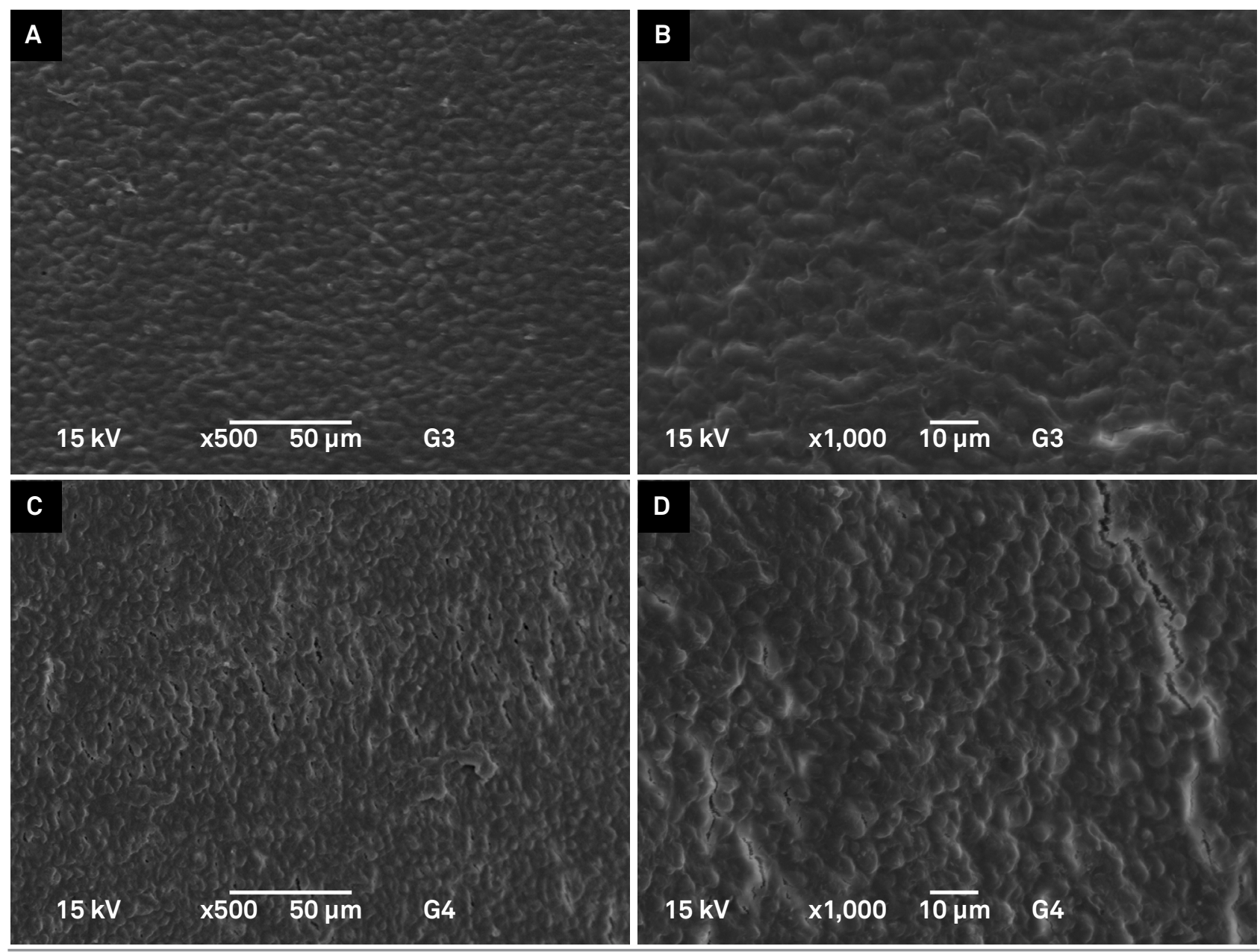

Figure 2. SEM representative image of samples from groups 3 ( $A$ and $B$ ) and 4 ( $C$ and $D$ ). In group 3 , the dentin tubules are not visible due to the deposition of minerals from the dentifrice. In $C$ and $D$, even though the laser has been used, the surfaces have the same characteristics as the samples from group 3. Magnification of $500 \times(A$ and $C)$ and $1000 \times$ and $(B$ and $D)$.

No photosensitizer was used in the present study, because it might impregnate the dentin after laser application and thus, compromise the aesthetics of the specimen.

A disadvantage of high intensity lasers for $\mathrm{DH}$ treatment is the possibility of some areas of dentin not receiving irradiation. In this study, the dentin was scanned in vertical and horizontal movements, with the tip of the optic fiber almost touching the hard tissue. While it is possible that irradiation performed with a $400 \mu \mathrm{m}$-diameter fiber might not reach some areas of the dentin, this method was chosen to simulate a clinical situation. According to the present results, this had no influence on the data, since the dentin permeability of group 2 was lower than that of group 1 and similar to that of groups 3 and 4 .
Another method for treating dentin hypersensitivity is the use of abrasive dentifrices containing calcium and phosphate crystals, which can form a layer on the exposed dentin and block the dentinal tubules. The dentifrice used in the present study contains $\mathrm{PRO}-$ Argin and calcium carbonate. Arginine is a positively charged amino acid obtained through diet or urea, which contains high levels of calcium, oxygen and phosphorus. Arginine and calcium carbonate form a protective wall rich in calcium phosphate, in the open dentinal tubules, occluding them immediately and providing fast pain relief. This barrier remains intact even when the tooth surface is exposed to acids. ${ }^{23}$ Past studies have shown that both arginine and calcium carbonate are needed to occlude the tubules. ${ }^{23,24}$ According to the dentifrice manufacturer, 
only one application is needed to reduce pain levels and, for a long lasting relief, that dentifrice may be used daily for tooth brushing for up to 2 weeks. Past studies evaluated the clinical efficacy of this dentifrice in reducing dentin hypersensitivity. ${ }^{25,26}$ As reported before, the dentifrice can be used for in-office or at-home treatment (during brushing, as regular toothpaste). In both cases, the Elmex Sensitive was effective in reducing DH. However, it was suggested that the treatment looses effectiveness if the patient stops brushing with the dentifrice. ${ }^{27} \mathrm{On}$ the other hand, as the laser treatment changes the morphology of the irradiated surface, a longer-lasting effect is expected. For this reason, the association of the abrasive toothpaste and high intensity laser was evaluated. The combination of laser and dentifrice treatments resulted in an effective occlusion of the tubules, reducing dentinal permeability.

The treatments evaluated in this study were conducted during one session. Thus, whether a single treatment would reduce root dentin permeability over the long term, could not be assessed. Also, whether these changes in dentin can resist an acid challenge or toothbrushing, was not evaluated. In vivo studies should be performed with the same conditions used here to evaluate the effects on pain sensitivity of the patient with DH. Clinical studies have shown that irradiation of the exposed dentin with high intensity lasers is considered an effective

\section{References}

1. West N, Seong J, Davies M. Dentine hypersensitivity. Monogr Oral Sci. 2014;25:108-22. doi:10.1159/000360749

2. Sgolastra F, Petrucci A, Severino M, Gatto R, Monaco A. Lasers for the treatment of dentin hypersensitivity: a meta-analysis. J Dent Res. 2013;92(6):492-9. doi:10.1177/0022034513487212

3. Assis JS, Rodrigues LK, Fonteles CS, Colares RC, Souza AM, Santiago SL. Dentin hypersensitivity after treatment with desensitizing agents: a randomized, double-blind, split-mouth clinical trial. Braz Dent J. 2011;22(2):157-61. doi:10.1590/S0103-64402011000200012

4. Brännström M, Lindén LA, Aström A. The hydrodynamics of the dental tubule and of pulp fluid: a discussion of its significance in relation to dentinal sensitivity. Caries Res. 1967;1(4):310-7. doi:10.1159/000259530 treatment for $\mathrm{DH} .{ }^{13,28}$ According to these studies, laser treatment seems to be more effective and durable than using abrasive toothpastes, because it induces morphological changes to the dentin surface. Another advantage of laser therapy is the treatment duration, since it is applied over just one session, unlike abrasive toothpaste, which has to be continuously used to exert its effect. . $^{3,25,26,28}$

An important factor in the treatment of $\mathrm{DH}$ is the patient's awareness of the need to take precautions to prevent recurrence or the appearance of new lesions. The patient should be oriented regarding proper tooth brushing techniques, the toothpaste used, the intake of acidic foods and drinks, and the treatment of endogenous factors, such as gastroesophageal reflux, and parafunctional habits that can lead to the dentin exposure to the oral environment. It should be emphasized that all DH treatments have advantages and disadvantages, but if applied in combination and with oral health recommendations for the patient, treatments can result in better outcomes. ${ }^{1}$

\section{Conclusions}

Within the limitations of the present study, it was concluded that the high intensity diode laser and the Elmex Sensitive Professional toothpaste were both effective in reducing dentin permeability, and the combination of the two treatments showed similar results than either laser or dentifrice alone.

5. González-Cabezas C, Hara AT, Hefferren J, Lippert F.

Abrasivity testing of dentifrices - challenges and current state of the art. Monogr Oral Sci. 2013;23:100-7. doi:10.1159/000350476

6. Hegde S, Rao BH, Kakar RC, Kakar A. A comparison of dentifrices for clinical relief from dentin hypersensitivity using the Jay Sensitivity Sensor Probe. Am J Dent. 2013;26 Spec No B:29B-36.

7. He T, Barker ML, Biesbrock AR, Miner M, Qaqish J, Sharma N. A clinical study to assess the effect of a stabilized stannous fluoride dentifrice on hypersensitivity relative to a marketed sodium fluoride/triclosan control. J Clin Dent. 2014;25(2):13-8.

8. Acharya AB, Surve SM, Thakur SL. A clinical study of the effect of calcium sodium phosphosilicate on dentin hypersensitivity. J Clin Exp Dent. 2013;5(1):e18-22. doi:10.4317/jced.50955 
9. Liu $\mathrm{H}, \mathrm{Hu}$ D. Efficacy of a commercial dentifrice containing $2 \%$ strontium chloride and $5 \%$ potassium nitrate for dentin hypersensitivity: a 3-day clinical study in adults in China. Clin Ther. 2012;34(3):614-22. doi:10.1016/j.clinthera.2012.01.027

10. Burwell AK, Litkowski LJ, Greenspan DC. Calcium sodium phosphosilicate (NovaMin): remineralization potential. Adv Dent Res. 2009;21(1):35-9. doi:10.1177/0895937409335621

11. Ladalardo TC, Pinheiro A, Campos RA, Brugnera Júnior A, Zanin F, Albernaz PL et al.. Laser therapy in the treatment of dentine hypersensitivity. Braz Dent J. 2004;15(2):144-50. doi:10.1590/S0103-64402004000200011

12. Gholami GA, Fekrazad R, Esmaiel-Nejad A, Kalhori KA. An evaluation of the occluding effects of Er;Cr:YSGG, Nd:YAG, $\mathrm{CO}_{2}$ and diode lasers on dentinal tubules: a scanning electron microscope in vitro study. Photomed Laser Surg. 2011;29(2):115-21. doi:10.1089/pho.2009.2628

13. Hashim NT, Gasmalla BG, Sabahelkheir AH, Awooda AM. Effect of the clinical application of the diode laser $(810$ $\mathrm{nm}$ ) in the treatment of dentine hypersensitivity. BMC Res Notes. 2014;13;7:31

14. Liu Y, Gao J, Gao Y, Xu S, Zhan X, Wu B. In vitro study of dentin Hypersensitivity treated by $980-\mathrm{nm}$ diode laser. J Lasers Med Sci. 2013;4(3):111-9.

15. Rizzante FA, Maenosono RM, Duarte MA, Furuse AY, Palma-Dibb RG, Ishikiriama SK. In Vitro evaluation of dentin hydraulic conductance after $980 \mathrm{~nm}$ diode laser irradiation. J Periodontol. 2016;87(3):320-6. doi:10.1902/jop.2015.150444

16. Toro CV, Derceli JR, Faraoni-Romano JJ, Marchi P, Pécora JD, Palma-Dibb RG. The use of an Er:YAG laser to remove demineralized dentin and its influence on dentin permeability. Microsc Res Tech. 2013;76(3):225-30. doi:10.1002/jemt.22156

17. Dall'Orologio GD, Ishihata H, Finger WJ, Sasaki K. In vitro and in vivo evaluation of the effectiveness of three dentin desensitizing treatment regimens. Am J Dent. 2014;27(3):139-44.

18. Farooq I, Moheet IA, AlShwaimi E. In vitro dentin tubule occlusion and remineralization competence of various toothpastes. Arch Oral Biol. 2015;60(9):1246-53. doi:10.1016/j.archoralbio.2015.05.012
19. Guedes AP, Moda MD, Suzuki TY, Godas AG, Sundfeld $\mathrm{RH}$, Briso AL et al. Effect of Fluoride-Releasing Adhesive Systems on the Mechanical Properties of Eroded Dentin. Braz Dent J. 2016;27(2):153-9. doi:10.1590/0103-644020160635

20. Umana M, Heysselaer D, Tielemans M, Compere P, Zeinoun T, Nammour S. Dentinal tubules sealing by means of diode lasers ( 810 and $980 \mathrm{~nm})$ : a preliminary in vitro study. Photomed Laser Surg. 2013;31(7):307-14. doi:10.1089/pho.2012.3443

21. Geraldo-Martins VR, Tanji EY, Wetter NU, Nogueira RD, Eduardo CP. Intrapulpal temperature during preparation with the Er:YAG laser: an in vitro study. Photomed Laser Surg. 2005;23(2):182-6. doi:10.1089/pho.2005.23.182

22. Secilmis A, Bulbul M, Sari T, Usumez A. Effects of different dentin thicknesses and air cooling on pulpal temperature rise during laser welding. Lasers Med Sci. 2013;28(1):167-70. doi:10.1007/s10103-012-1108-1

23. Markowitz K. A new treatment alternative for sensitive teeth: a desensitizing oral rinse. J Dent. 2013;41 Suppl 1:S1-11. doi:10.1016/j.jdent.2012.09.007

24. Collins JR, Richardson D, Sotero K, Mateo LR, Mauriz I. Beneficial effects of an arginine-calcium carbonate desensitizing paste for treatment of dentin hypersensitivity. Am J Dent. 2013;26(2):63-7.

25. Hamlin D, Mateo LR, Dibart S, Delgado E, Zhang YP, DeVizio W. Comparative efficacy of two treatment regimens combining in-office and at-home programs for dentin hypersensitivity relief: a 24-week clinical study. Am J Dent. 2012;25(3):146-52.

26. Kapferer I, Pflug C, Kisielewsky I, Giesinger J, Beier US, Dumfahrt H. Instant dentin hypersensitivity relief of a single topical application of an in-office desensitizing paste containing $8 \%$ arginine and calcium carbonate: a split-mouth, randomized-controlled study. Acta Odontol Scand. 2013;71(3-4):994-9. doi:10.3109/00016357.2012.741701

27. Chu CH, Lo EC. Immediate post-application effect of professional prophylaxis with $8 \%$ arginine-calcium carbonate desensitizing paste on hypersensitive teeth. A practitioner-based clinical trial. Am J Dent. 2014;27(1):7-11.

28. Raichur PS, Setty SB, Thakur SL. Comparative evaluation of diode laser, stannous fluoride gel, and potassium nitrate gel in the treatment of dentinal hypersensitivity. Gen Dent. 2013;61(3):66-71. 